This item was submitted to Loughborough's Research Repository by the author.

Items in Figshare are protected by copyright, with all rights reserved, unless otherwise indicated.

\title{
Hydro-acoustic frequencies of the weakly compressible mild-slope equation
}

\section{PLEASE CITE THE PUBLISHED VERSION}

https://doi.org/10.1017/jfm.2016.791

\section{PUBLISHER}

(c) Cambridge University Press

\section{VERSION}

AM (Accepted Manuscript)

\section{PUBLISHER STATEMENT}

This work is made available according to the conditions of the Creative Commons Attribution-NonCommercialNoDerivatives 4.0 International (CC BY-NC-ND 4.0) licence. Full details of this licence are available at: https://creativecommons.org/licenses/by-nc-nd/4.0/

\section{LICENCE}

CC BY-NC-ND 4.0

\section{REPOSITORY RECORD}

Renzi, Emiliano. 2016. "Hydro-acoustic Frequencies of the Weakly Compressible Mild-slope Equation". Loughborough University. https://hdl.handle.net/2134/23262. 


\title{
Hydro-acoustic frequencies of the weakly compressible mild-slope equation
}

\author{
Emiliano Renzi ${ }^{1} \dagger$ \\ ${ }^{1}$ Department of Mathematical Sciences, Loughborough University, Loughborough, Leics LE11 \\ 3TU, UK \\ (Received $\mathrm{xx}$; revised $\mathrm{xx}$; accepted $\mathrm{xx}$ )
}

We present a novel analytical solution for hydro-acoustic waves in a weakly compressible fluid over a slowly varying bottom. Application of a multiple-scale perturbation technique and matched asymptotic analysis leads to a uniform analytical solution of the depthaveraged governing equations in three dimensions. We show that the slow depth variation plays a leading-order effect on the evolution of the normal mode amplitude and direction. This dynamics is much richer than the two-dimensional limit analysed in previous studies. For tsunamigenic disturbances, we show that the hydro-acoustic wave field is made up by longshore trapped and offshore propagating components, which were not explicated in previous work. For a plane beach, we find an exact analytical solution of the model equation in terms of integrals of Bessel functions. Our model offers a physical insight into the evolution of hydro-acoustic waves of interest for the design of tsunami early warning systems.

\section{Key words:}

\section{Introduction}

A systematic investigation on the mechanisms of co-generation of gravity and hydroacoustic (HA) waves by the same source has started only very recently, motivated by interesting possibilities of application in coastal engineering. Stiassnie (2010) was among the first to derive an analytical model of gravity and HA wave generation by a tsunamigenic bottom deformation in uniform water depth. Later, Renzi \& Dias (2014a) solved a similar problem, but with the forcing term being a localised surface pressure perturbation in space and time. The propagation of HA waves generated by bottom movements over a range dependent environment is a more complex problem. Kadri \& Stiassnie (2013) and Kadri (2015) recently developed a non-uniform asymptotic approximation for shoaling HA waves over a slowly varying bottom in two dimensions (2D). However, Kadri \& Stiassnie (2013) and Kadri (2015)'s results cannot be applied to a 3D geometry, where the combined effect of shoaling and refraction dramatically alters the propagation dynamics with respect to the $2 \mathrm{D}$ scenario. A significant advancement in the field is represented by the seminal work of Sammarco et al. (2013), who for the first time derived a 3D form of the mild-slope equation for weakly compressible fluids (MSEWC) and solved it numerically. Sammarco et al. (2013)'s numerical solution of the model equation, however, does not discriminate explicitly between the eigenfrequencies of the HA modes and how they transform as they propagate.

$\dagger$ Email address for correspondence: E.Renzi@lboro.ac.uk 
In this paper, we derive a novel analytical solution for the propagation of hydro-acoustic (HA) wave frequencies, generated by bottom movements, in a 3D domain occupied by a compressible fluid over a range dependent environment. The paper aims to: (i) review Sammarco et al. (2013)'s formulation of the mild-slope equation for compressible fluids to derive a form that is solvable analytically in a 3D domain (see $\S 2$ ), (ii) obtain a new analytical solution of the model equation (see $\S 3$ ) and (iii) identify the physical nature of the different kinds of HA frequencies excited by tsunamigenic disturbances over a non-uniform bottom in 3D (see $\S 4)$.

\section{Mathematical model}

\subsection{Model equations}

Calling on Sammarco et al. (2013), consider the motion of a slightly compressible fluid of density $\rho=\rho_{0}+\rho^{\prime}$, where $\rho_{0}$ is the constant ambient density and $\rho^{\prime} \ll \rho_{0}$ is a small perturbation due to compressibility. Let $(\boldsymbol{x}, z)=(x, y, z)$ describe the coordinate of a point in a three-dimensional fluid domain $\mathcal{D}$, with the $z$ axis orthogonal to the horizontal $(x, y)$ plane and pointing upwards from the unperturbed water level $z=0$. The bottom of the ocean is at $z=-h(\boldsymbol{x})+H(\boldsymbol{x}, t)$, where $h(\boldsymbol{x})$ is a fixed impermeable surface and $H(\boldsymbol{x}, t)$ a prescribed time-dependent seafloor motion; $t$ denotes time. The boundary-value problem for a weakly compressible, inviscid fluid and irrotational motion is:

$$
\begin{aligned}
\Phi_{t t}=c^{2}\left(\nabla^{2} \Phi+\Phi_{z z}\right), & (\boldsymbol{x}, z) \in \mathcal{D} \\
\Phi_{t t}+g \Phi_{z}=0, & z=0 \\
\Phi_{z}+\nabla h \cdot \nabla \Phi=-H_{t}, & z=-h(\boldsymbol{x}) .
\end{aligned}
$$

In the latter system of equations, $\Phi(\boldsymbol{x}, z, t)$ is the $3 \mathrm{D}$ velocity potential, $g$ is the acceleration due to gravity and $c=1480 \mathrm{~m} / \mathrm{s}$ is the speed of sound in water, assumed constant. Also, $\nabla f(\boldsymbol{x})=\left(f_{x}, f_{y}\right)$ is the $2 \mathrm{D}$ gradient and subscripts denote differentiation with respect to the relevant variable. The system (2.1)-(2.3) describes acoustic-gravity waves generated by the bottom disturbance $H$ and is the same as that considered by Sammarco et al. (2013). In this paper we shall develop a mathematical method to solve the system of governing equations (2.1)-(2.3) analytically. First, we shall adopt a Galerkin approach (Massel 2013) to derive the governing partial differential equation (PDE) in the horizontal plane. Then we shall solve it by combining a multiple-scale perturbation approach with asymptotic analysis. We formulate the mathematical problem for a generic single frequency $f$ of the forcing spectrum, assuming the decompositions:

$$
H(\boldsymbol{x}, t)=\operatorname{Re}\left\{\bar{H}(\boldsymbol{x}) \mathrm{e}^{-\mathrm{i} \omega t}\right\}, \quad \Phi(\boldsymbol{x}, z, t)=\operatorname{Re}\left\{\bar{\Phi}(\boldsymbol{x}, z) \mathrm{e}^{-\mathrm{i} \omega t}\right\},
$$

where $\mathrm{i}$ is the imaginary unit and $\omega=2 \pi f$ is the angular frequency. $\operatorname{Re}\{\cdot\}$ denotes the real part and will be omitted in the following for the sake of brevity. Results for generic time-dependent disturbances can be found by Fourier superposition of the harmonic solution $\Phi$ (Mei et al. 2005; Sammarco et al. 2013). Using (2.4), the system (2.1)-(2.3) becomes

$$
\begin{gathered}
\left(\nabla^{2}+\frac{\omega^{2}}{c^{2}}\right) \bar{\Phi}+\bar{\Phi}_{z z}=0, \quad(\boldsymbol{x}, z) \in \mathcal{D} \\
\bar{\Phi}_{z}-\frac{\omega^{2}}{g} \bar{\Phi}=0, \quad z=0 \\
\bar{\Phi}_{z}+\nabla h \cdot \nabla \bar{\Phi}=\mathrm{i} \omega \bar{H}, \quad z=-h(\boldsymbol{x}),
\end{gathered}
$$


where the complex potential $\bar{\Phi}(\boldsymbol{x}, z)$ must be bounded in the fluid domain $\mathcal{D}$. Now expand the spatial potential into a Galerkin series (Massel 2013)

$$
\bar{\Phi}(\boldsymbol{x}, z)=\sum_{n=0}^{\infty} \bar{\Phi}_{n}(\boldsymbol{x}, z)=\sum_{n=0}^{\infty} \phi_{n}(\boldsymbol{x}) Z_{n}(\boldsymbol{x}, z),
$$

where

$$
Z_{n}(\boldsymbol{x}, z)=\frac{\sqrt{2} \cosh \left[\beta_{n}(z+h)\right]}{\left[h+\frac{g}{\omega^{2}} \sinh ^{2}\left(\beta_{n} h\right)\right]^{1 / 2}} .
$$

In eq. (2.9) the $\beta_{n}=\beta_{n}[h(\boldsymbol{x})]$ are the solutions of the dispersion relation:

$$
\begin{aligned}
& \omega^{2}=g \beta_{0} \tanh \left(\beta_{0} h\right), \quad n=0, \\
& \beta_{n}=\mathrm{i} \tilde{\beta}_{n}, \quad \omega^{2}=-g \tilde{\beta}_{n} \tan \left(\tilde{\beta}_{n} h\right), \quad n>0 .
\end{aligned}
$$

The 0th mode represents the surface gravity wave, which is hardly influenced by compressibility (Yamamoto 1982; Stiassnie 2010; Renzi \& Dias 2014a). The higher modes $n>0$ represent the associated HA waves, which for given frequency $\omega$ can be either evanescent or propagating at large $|\boldsymbol{x}|$, depending on the system parameters (see $\S 3$ ). Now introduce the inner product

$$
\langle f(z), g(z)\rangle=\int_{-h(\boldsymbol{x})}^{0} f(z) g(z) \mathrm{d} z,
$$

which together with $(2.10)-(2.11)$ yields $\left\langle Z_{n}, Z_{m}\right\rangle=\delta_{n m}$. Hence the $Z_{n}$ are a set of orthonormal eigenfunctions in $(-h(\boldsymbol{x}), 0)$. The eigenfunctions $(2.9)$ are a normalised form of those used in the solution of Sammarco et al. (2013), namely $f_{n}=\cosh \left[\beta_{n}(z+\right.$ $h)] / \cosh \left(\beta_{n} h\right)$. Note that the latter expression becomes singular for $z<0$ in the limit $\beta_{n} \rightarrow \mathrm{i}(2 n-1) \pi /(2 h)$, which describes the HA modes of the system (Yamamoto 1982; Stiassnie 2010). Such a singularity would prevent us from obtaining an analytical representation of the HA field quantities (though a numerical solution is still possible, see Sammarco et al. 2013). Conversely, the normalised eigenfunctions (2.9) allow us to solve the problem analytically. Now, let us expand $\bar{H}$ into a Galerkin series too

$$
\bar{H}(\boldsymbol{x})=\sum_{n=0}^{\infty} \bar{H}_{n}(\boldsymbol{x})=\sum_{n=0}^{\infty} H_{n}(\boldsymbol{x}) Z_{n}(\boldsymbol{x}, z),
$$

where the expansion terms

$$
H_{n}(\boldsymbol{x})=\bar{H}(\boldsymbol{x}) \frac{2 \sinh \left(\beta_{n} h\right)}{\left[2 \beta_{n}^{2} h+\beta_{n} \sinh \left(2 \beta_{n} h\right)\right]^{1 / 2}}
$$

follow from the orthogonality of the $Z_{n}$ (2.9). We are now in a position to derive a depth-averaged form for the boundary-value problem (2.5)-(2.7). By following the depthaveraged approach of Sammarco et al. (2013), but using the orthonormal eigenfunctions (2.9), we get a normalised version of the mild-slope equation for weakly compressible fluids (MSEWC):

$$
\nabla^{2} \phi_{n}+\left(\frac{\omega^{2}}{c^{2}}+\beta_{n}^{2}\right) \phi_{n}=\mathrm{i} \omega \gamma_{n} H_{n}, \quad n=0,1, \ldots
$$

where

$$
\gamma_{n}(\boldsymbol{x})=\frac{4 \beta_{n}}{2 \beta_{n} h+\sinh \left(2 \beta_{n} h\right)}
$$


has the dimension of a wavenumber. The 0th mode of (2.14) represents a progressive acoustic-gravity wave (Sammarco et al. 2013). Due to the large difference between gravity and HA wave propagation speed in water, the gravity mode $n=0$ is hardly influenced by compressibility (Stiassnie 2010). Numerous analytical and numerical models for the gravity mode propagation over slowly varying bottom are already available in the literature (e.g Mei et al. 2005; Massel 2013) and will not be discussed further. Here we shall focus on the HA modes $n \geqslant 1$ of $(2.14)$.

\subsection{The MSE for $H A$ waves}

As anticipated in $\S 2.1$, a good approximation for the HA wavenumbers $\beta_{n}=\mathrm{i} \tilde{\beta}_{n}$ is

$$
\tilde{\beta}_{n}[h(\boldsymbol{x})]=\frac{(2 n-1) \pi}{2 h(\boldsymbol{x})},
$$

see for example Yamamoto (1982), Stiassnie (2010) and Renzi \& Dias (2014a). As a consequence, the eigenmodes (2.9) become

$$
Z_{n}(\boldsymbol{x}, z)=\sqrt{\frac{2}{h}} \cos \left[(2 n-1) \frac{\pi}{2}\left(1+\frac{z}{h(\boldsymbol{x})}\right)\right],
$$

the forcing terms (2.13) become

$$
H_{n}(\boldsymbol{x})=\frac{(-1)^{n+1}}{\left(n-\frac{1}{2}\right) \pi} \sqrt{2 h(\boldsymbol{x})} \bar{H}(\boldsymbol{x})
$$

and (2.15) simplifies to $\gamma_{n}(\boldsymbol{x})=2 / h(\boldsymbol{x})$. Substituting the latter expressions into (2.14), one obtains the sought equation for the HA modes:

$$
\nabla^{2} \phi_{n}+k_{n}^{2}(\boldsymbol{x}) \phi_{n}=-\frac{4}{\pi} \frac{(-1)^{n}}{2 n-1} \mathrm{i} \omega \bar{H}(\boldsymbol{x}) \sqrt{\frac{2}{h(\boldsymbol{x})}}, \quad n=1,2, \ldots,
$$

where

$$
k_{n}(\boldsymbol{x})=\sqrt{\frac{\omega^{2}}{c^{2}}-\frac{(2 n-1)^{2} \pi^{2}}{4 h^{2}(\boldsymbol{x})}}
$$

is the horizontal index of refraction of the $n$th HA mode. Expression (2.19) is a specific form of the normalised MSEWC. We name (2.19) the MSE for HA waves (MSEHA).

\section{Free hydro-acoustic waves over topography}

\subsection{General solution}

In this section we study the homogeneous form of the MSEHA (2.19), which describes free incoming and outgoing HA waves, in the absence of tsunamigenic forcing. From now on we consider a generic HA mode, say $n \in \mathbb{N}$, on a slowly varying topography in the two horizontal dimensions ( $2 \mathrm{HD}), h=h(\epsilon \boldsymbol{x}), \epsilon \ll 1$. We shall now derive a multiple-scale approximation of the spatial HA potential $\phi_{n}(\boldsymbol{x})$. Introduction of the slow variables

$$
\xi=\epsilon x, \quad \eta=\epsilon y,
$$

transforms the homogeneous version of (2.19) into

$$
\epsilon^{2} \tilde{\nabla}^{2} \phi_{n}+k_{n}^{2}(\xi, \eta) \phi_{n}=0,
$$

where $\tilde{\nabla}=(\partial / \partial \xi, \partial / \partial \eta)$ is the nabla operator in the slow coordinates. We will first consider the case of real $k_{n}$ and deal with complex wavenumbers later on. Note that, in 
the much simpler $1 \mathrm{D}$ case over a flat bottom, where $k_{n}=$ const, the spatial potential would be a combination of $\phi_{n}(\xi)=\exp \left( \pm \mathrm{i} k_{n} \xi / \epsilon\right)$, see e.g. Jensen et al. (2011). For a $2 \mathrm{HD}$ varying bathymetry, by similarity we look for solutions of the form

$$
\phi_{n}(\xi, \eta)=A_{n}(\xi, \eta) \exp \left[\frac{\mathrm{i}}{\epsilon} S_{n}(\xi, \eta)\right],
$$

where $A_{n}(\xi, \eta)$ and $S_{n}(\xi, \eta)$ are unknown real amplitude and phase functions, respectively. Equation (3.3) is a multiple-scale WKB expression, in which the wave amplitude varies with the slow coordinates $\xi$ and $\eta$, while the phase varies with the fast coordinates $(\xi, \eta) \epsilon^{-1}$, see Bender \& Orszag (1999). Substituting (3.3) into (3.2) and taking the real and imaginary parts of (3.2) separately zero, we get a system of two nonlinear PDEs:

$$
\begin{gathered}
\epsilon^{2} \tilde{\nabla}^{2} A_{n}-\left|\tilde{\nabla} S_{n}\right|^{2} A_{n}+k_{n}^{2} A_{n}=0, \\
2 \tilde{\nabla} A_{n} \cdot \tilde{\nabla} S_{n}+A_{n} \tilde{\nabla}^{2} S_{n}=0 .
\end{gathered}
$$

Note that the original equation (3.2) and the system (3.4)-(3.5) are absolutely equivalent, for only the substitution (3.3) has been made so far. Let us now expand the amplitude and phase functions, respectively

$$
A_{n}(\xi, \eta)=A_{n}^{(0)}(\xi, \eta)+\epsilon A_{n}^{(1)}(\xi, \eta)+O\left(\epsilon^{2}\right)
$$

and

$$
S_{n}(\xi, \eta)=S_{n}^{(0)}(\xi, \eta)+\epsilon S_{n}^{(1)}(\xi, \eta)+O\left(\epsilon^{2}\right),
$$

where $\epsilon \ll 1$. Substitution of (3.6)-(3.7) into the system (3.4)-(3.5) yields, respectively, an eikonal equation

$$
\left|\tilde{\nabla} S_{n}^{(0)}(\xi, \eta)\right|^{2}=k_{n}^{2}(\xi, \eta)
$$

and a transport equation

$$
\tilde{\nabla} \cdot\left\{\left[A_{n}^{(0)}(\xi, \eta)\right]^{2} \tilde{\nabla} S_{n}^{(0)}(\xi, \eta)\right\}=0,
$$

at the order $O(1)$. The eikonal equation (3.8) can be solved numerically to obtain $S_{n}^{(0)}$ for given $h(\epsilon \boldsymbol{x})$, see Dingemans (1997); Mei et al. (2005). Once the phase function is known, then the amplitude $A_{n}^{(0)}$ can be found by solving the transport equation (3.9), so that the spatial potential $\phi_{n}(3.3)$ is determined with an error $O(\epsilon)$, for $\epsilon \ll 1$. This numerical strategy can be applied to higher orders of $\epsilon$, for any slowly varying bottom profile.

In the following, based on the decomposition (3.3), the eikonal equation (3.8) and the transport equation (3.9), we shall first prove that the MSEHA (2.19) conserves the energy flux over a slowly varying depth. Based on this result, we shall derive a uniform analytical solution for the case of parallel iso-baths in $\S 3.2$.

\subsubsection{Conservation of energy}

The energy flux across a vertical cross section of unit width orthogonal to the direction of propagation of the $n$th HA mode is given in physical variables by

$$
\mathcal{F}_{n}(\boldsymbol{x})=\frac{\rho \omega}{2} \operatorname{Im}\left\{\phi_{n}^{*} \nabla \phi_{n}\right\}
$$

where $\operatorname{Im}\{\cdot\}$ denotes the imaginary part and $\phi_{n}^{*}$ is the complex conjugate of $\phi_{n}$, see Appendix A. Consider a slowly varying bottom in $2 \mathrm{HD}, h=h(\epsilon \boldsymbol{x})$, with $\epsilon \ll 1$. 
Substitution of the slow variables (3.1) and use of the expansions (3.3) and (3.6)-(3.7) transforms (3.10) into

$$
\mathcal{F}_{n}(\xi, \eta)=\frac{\rho \omega}{2}\left[A_{n}^{(0)}(\xi, \eta)\right]^{2} \tilde{\nabla} S_{n}^{(0)}(\xi, \eta)
$$

Multiplying the latter expression by $2 /(\rho \omega)$ and taking the divergence, we obtain precisely the transport equation (3.9), which then yields

$$
\tilde{\nabla} \cdot \mathcal{F}_{n}=0
$$

i.e. a conservation equation (Mei et al. 2005). Expression (3.11) proves that the MSEHA (2.19) conserves the energy flux over a $2 \mathrm{HD}$ slowly varying bottom. Note that we do not need to apply auxiliary conditions on wave energy conservation to solve the MSEHA, as its solutions already satisfy the conservation law (3.11).

\subsection{Solution for cylindrical topography}

We shall now consider the case of straight and parallel iso-baths, $h=h(\epsilon x)$, which is amenable to analytical investigation. The depth contours are now parallel to the $y$ axis, while the $x$ axis points offshore. Using the slow variables $(\xi, \eta)$, see $(3.1)$, the free-wave solutions of the MSEHA (2.19) have the form (3.3), where the amplitude function $A_{n}$ and the phase function $S_{n}$ must satisfy the system of PDEs (3.8)-(3.9) for $\epsilon \ll 1$; the superscripts are dropped for simplicity. In the case of straight and parallel iso-baths, the horizontal index of refraction $k_{n}(2.20)$ is a function of the offshore coordinate only, and the eikonal equation (3.8) yields the nonlinear first-order PDE

$$
S_{n_{\xi}}^{2}+S_{n_{\eta}}^{2}=k_{n}^{2}(\xi)
$$

while the transport equation (3.9) becomes

$$
2 \tilde{\nabla} A_{n} \cdot \tilde{\nabla} S_{n}+A_{n} \tilde{\nabla}^{2} S_{n}=0 .
$$

Let us first consider the eikonal equation (3.12). Define the wavenumber vector $\boldsymbol{k}_{n}(\xi)=$ $k_{n}(\xi)\left\{\cos \theta_{n}, \sin \theta_{n}\right\}$, where $\theta_{n}=\theta_{n}(\xi)$ is the angle between the $\boldsymbol{k}_{n}$ vector and the $\xi$ axis, still unknown. Then (3.12) is equivalent to the vector identity

$$
\tilde{\nabla} S_{n}=\boldsymbol{k}_{n}
$$

which shows that the wavenumber vector $\boldsymbol{k}_{n}$ is orthogonal to the lines of constant phase $S_{n}$, i.e. the HA wave crests. Now, the curl of (3.14) gives $0=\tilde{\nabla} \times \tilde{\nabla} S_{n}=\left(k_{n} \sin \theta_{n}\right)_{\xi}$, which upon integration yields

$$
k_{n}(\xi) \sin \left[\theta_{n}(\xi)\right]=\alpha_{n},
$$

where $\alpha_{n}$ is a real constant. Expression (3.15) shows that the longshore component of $\boldsymbol{k}_{n}$ keeps constant over straight and parallel iso-baths. That is an extension to HA waves of Snell's law for gravity waves (Mei et al. 2005). Substitution of (3.15) into the eikonal equation (3.12) and further integration finally yield the phase function

$$
S_{n}(\xi, \eta)= \pm \int_{\xi_{0}}^{\xi} \mu_{n}(\sigma) \mathrm{d} \sigma+\alpha_{n} \eta,
$$

where $\xi_{0}$ is an arbitrary point in the horizontal domain and

$$
\mu_{n}(\xi)=\mu_{n}[h(\xi)]=\sqrt{\frac{\omega^{2}}{c^{2}}-\frac{(2 n-1)^{2} \pi^{2}}{4 h^{2}(\xi)}-\alpha_{n}^{2}}
$$


is the offshore component of the wavenumber. In fact, substituting (3.16) back into (3.14), the wavenumber vector becomes

$$
\boldsymbol{k}_{n}=\boldsymbol{k}_{n}^{ \pm}(\xi)=\left\{ \pm \mu_{n}(\xi), \alpha_{n}\right\}
$$

where the top sign $(+)$ refers to outgoing HA waves and the bottom sign $(-)$ refers to incoming HA waves. As a consequence of (3.18), the orientation of the wavenumber vector with respect to the offshore direction is given by

$$
\theta_{n}^{+}=\arctan \left(\frac{\alpha_{n}}{\mu_{n}}\right), \quad \theta_{n}^{-}=\pi-\arctan \left(\frac{\alpha_{n}}{\mu_{n}}\right),
$$

for the outgoing and incoming waves, respectively. We are now in a position to solve the transport equation (3.13) for the amplitude function $A_{n}$. Substituting (3.16) into (3.13), we get two first-order PDEs:

$$
\pm 2 \mu_{n} A_{n_{\xi}}+2 \alpha_{n} A_{n_{\eta}}=\mp A_{n} \mu_{n_{\xi}},
$$

one for each sign of $S_{n}$ in (3.16). Expression (3.20) can be solved with the method of characteristics (Hildebrand 1976). The general solution of (3.20) is of the form $v=f(u)$. In the latter, $u\left(\xi, \eta, A_{n}\right)=c_{1}$ and $v\left(\xi, \eta, A_{n}\right)=c_{2}$ are the general solutions of

$$
\pm \frac{\mathrm{d} \xi}{2 \mu_{n}(\xi)}=\frac{\mathrm{d} \eta}{2 \alpha_{n}}
$$

and

$$
\frac{\mathrm{d} \xi}{2 \mu_{n}(\xi)}=-\frac{\mathrm{d} A_{n}}{A_{n} \mu_{n_{\xi}}(\xi)},
$$

respectively; $c_{1}$ and $c_{2}$ are arbitrary integration constants. Solving (3.21) by parts gives

$$
u(\xi, \eta)=-\eta \pm \alpha_{n} \int \frac{\mathrm{d} \xi}{\mu_{n}(\xi)}=c_{1},
$$

while the solution of $(3.22)$ is

$$
v\left(\xi, \eta, A_{n}\right)=A_{n}(\xi, \eta) \mu_{n}^{1 / 2}=c_{2} .
$$

Hence $v=f(u)$ yields

$$
A_{n}=A_{n}^{ \pm}(\xi, \eta)=\mu_{n}^{-1 / 2}(\xi) f\left( \pm \int \frac{\alpha_{n}}{\mu_{n}(\xi)} \mathrm{d} \xi-\eta\right),
$$

where $f$ is an arbitrary function and again the + sign denotes outgoing waves, while the - sign denotes incoming waves. On a cylindrical topography, the amplitude function must satisfy $A_{n_{\eta}}=0$, see Mei et al. (2005). This implies that the arbitrary function $f$ in (3.23) must satisfy

$$
f\left(-\int \frac{\alpha_{n}}{\mu_{n}(\xi)} \mathrm{d} \xi-\eta\right)=a_{n}
$$

for the incoming wave and

$$
f\left(\int \frac{\alpha_{n}}{\mu_{n}(\xi)} \mathrm{d} \xi-\eta\right)=b_{n}
$$

for the outgoing wave. In (3.24)-(3.25), $a_{n}$ and $b_{n}$ are two arbitrary integration constants, depending on the boundary conditions of the problem at $\xi=\xi_{0}$. Now recall that in this section we are considering free waves, as we have eliminated the localised forcing term in (2.19). In the absence of localised forcing, no radiation condition can be applied (Mei 
et al. 2005). Hence, we must retain both incoming and outgoing waves in the free-wave solution. Now substitute the phase function (3.16) and the amplitude function (3.23), together with (3.24)-(3.25), into expression (3.3) for the spatial potential $\phi_{n}$. Then sum both incoming and outgoing waves, and revert to the physical variables (3.1) to obtain the spatial HA potential

$$
\begin{aligned}
\phi_{n}(x, y)=\frac{1}{\sqrt{\mu_{n}(\epsilon x)}} & \left\{a_{n} \exp \left[-\mathrm{i}\left(\int_{x_{0}}^{x} \mu_{n}(\epsilon s) \mathrm{d} s-\alpha_{n} y\right)\right]\right. \\
& \left.+b_{n} \exp \left[\mathrm{i}\left(\int_{x_{0}}^{x} \mu_{n}(\epsilon s) \mathrm{d} s+\alpha_{n} y\right)\right]\right\} .
\end{aligned}
$$

The latter is a multiple-scale approximation of the solution (Bender \& Orszag 1999). Along the longshore direction $y,(3.26)$ oscillates with a constant wavenumber $\alpha_{n}$. Along the offshore direction $x$, (3.26) describes waves of slowly varying amplitude and fast varying phase. Now note that, upon certain combinations of $\omega$ and $\alpha_{n}$, the offshore wavenumber $\mu_{n}(3.17)$ can become purely imaginary, i.e. $\mu_{n}^{2}<0$. In such a case, the HA perturbation (3.26) ceases to propagate along $x$. This means that the physical behaviour of each HA mode $\phi_{n}$ is governed by the sign of $\mu_{n}^{2}(\epsilon x)$. For any $\omega>0$ we distinguish the following cases.

(1): $\left|\alpha_{n}\right| \geqslant \omega / c$. Then $\mu_{n}^{2}<0$ for all $h$ and the potential $\phi_{n}$ (3.26) is a decaying exponential along $x$ :

$$
\phi_{n}(x, y)=\left\{\begin{array}{cc}
\frac{a_{n}}{\sqrt{\tilde{\mu}_{n}(\epsilon x)}} \exp \left(\int_{x_{0}}^{x} \tilde{\mu}_{n}(\epsilon s) \mathrm{d} s\right) \exp \left(\mathrm{i} \alpha_{n} y\right) & x<x_{0} \\
\frac{b_{n}}{\sqrt{\tilde{\mu}_{n}(\epsilon x)}} \exp \left(-\int_{x_{0}}^{x} \tilde{\mu}_{n}(\epsilon s) \mathrm{d} s\right) \exp \left(\mathrm{i} \alpha_{n} y\right) & x>x_{0}
\end{array},\right.
$$

where

$$
\tilde{\mu}_{n}(\epsilon x)=\sqrt{\frac{(2 n-1)^{2} \pi^{2}}{4 h^{2}(\epsilon x)}+\alpha_{n}^{2}-\frac{\omega^{2}}{c^{2}}} .
$$

The wave field (3.27) still propagates along $y$, i.e. parallel to bathymetry, but decays along $x$ regardless of the water depth! This is different from the known dynamics in $2 \mathrm{D}$, where each $n$th HA mode decays only in regions where the water depth is less than the cut-off value $(2 n-1) \pi c /(2 \omega)$, see Yamamoto (1982); Stiassnie (2010); Kadri \& Stiassnie (2013); Kadri (2015). The analytical structure of (3.27) reveals that no simple harmonic HA waves can exist when $\left|\alpha_{n}\right| \geqslant \omega / c$, i.e. all HA waves are trapped. Similar to trapped gravity waves over topography (see Mei et al. 2005), trapped HA waves cannot be excited by a simple harmonic incident HA wave with a linearised mechanism. However, external excitation of the trapped modes (3.27) is still possible by a localised source, such as a submarine failure. This case will be further analysed in $\S 4$.

(2): $\left|\alpha_{n}\right|<\omega / c$. In this case, the physical behaviour of $\phi_{n}$ depends on the water depth $h$. Let us first define the critical depth

$$
d_{n}=\frac{(2 n-1) \pi}{2 \sqrt{\frac{\omega^{2}}{c^{2}}-\alpha_{n}^{2}}},
$$

as the depth at which the offshore wavenumber $\mu_{n}=0$. Then (3.17) rewrites

$$
\mu_{n}(\epsilon x)=\sqrt{\left(\frac{\omega^{2}}{c^{2}}-\alpha_{n}^{2}\right)\left(1-\frac{d_{n}^{2}}{h^{2}(\epsilon x)}\right)},
$$

so that $\mu_{n}^{2}(\epsilon x) \lesseqgtr 0$ when $h(\epsilon x) \lesseqgtr d_{n}$. Mathematically, the points $x=x_{n}$ such that 
$h\left(\epsilon x_{n}\right)=d_{n}$ and, consequently, $\mu_{n}\left(\epsilon x_{n}\right)=0$ are turning points, through which the HA potential $\phi_{n}(3.26)$ turns from exponential $\left(\mu_{n}^{2}<0\right)$ to oscillatory $\left(\mu_{n}^{2}>0\right)$. For definiteness, let us consider a monotonically increasing bottom depth, $h_{x}>0$. Then there exists at the most one turning point $x_{n}$, solution of $h\left(\epsilon x_{n}\right)=d_{n}$, such that $\mu_{n}^{2} \lesseqgtr 0$ if $x \lesseqgtr x_{n}$. Note that (3.26) ceases to be valid in the limit $x \rightarrow x_{n}$ (i.e. $\mu_{n} \rightarrow 0$ ). We shall now determine a uniform asymptotic expansion of (3.26) through the turning point at $x=x_{n}$ (Bender \& Orszag 1999). The physical optics approximation (3.26) suggests that a uniform asymptotic solution of (2.19) must be of the form

$$
\phi_{n}(x, y)=X_{n}(x) \exp \left(\mathrm{i} \alpha_{n} y\right) .
$$

In the latter, $\alpha_{n}$ is still the constant longshore wavenumber following from Snell's law (3.15), and $X_{n}$ is the unknown offshore component of the HA potential. Substitution of (3.31) into the homogeneous form of (2.19) and use of the slow variables (3.1) yields

$$
\epsilon^{2} X_{n}^{\prime \prime}+\mu_{n}^{2}(\xi) X_{n}=0
$$

where the primes denote differentiation with respect to the slow variable $\xi$. Expression (3.32) has the form of a time-independent Schrödinger equation of one spatial dimension (Cheng 2007). Using the method of asymptotic matching at the turning point (Bender \& Orszag 1999), the details of which we omit for the sake of brevity, and then reverting to the physical variable $x=\xi / \epsilon$, the solution $X_{n}$ of (3.32) can be shown to admit the following uniform asymptotic expansion:

$$
X_{n}(x)=\frac{2 \sqrt{\pi} a_{n}}{\sqrt{\mu_{n}(\epsilon x)}}\left[F_{n}(x)\right]^{1 / 6} \mathrm{Ai}\left[-F_{n}^{2 / 3}(x)\right] .
$$

In the latter, $a_{n}$ is the HA wave amplitude, Ai is the Airy function and

$$
F_{n}(x)=\frac{3}{2} \int_{x_{n}}^{x} \mu_{n}(\epsilon s) \mathrm{d} s .
$$

Since $F_{n}(x)$ becomes purely imaginary if $x<x_{n}$, (3.33) requires the introduction of a branch-cut along the positive real axis to avoid multi-valuedness. The uniform asymptotic expansion for the HA potential $\phi_{n}$ is then found by substituting (3.33) into (3.31).

\subsubsection{Numerical example}

Let us now study the propagation of free travelling HA waves, incoming from the far field at $x \rightarrow \infty$, over a slowly varying bottom. A possible physical mechanism for the generation of an incoming HA wave of frequency $f$ is the far-field interaction of two gravity waves of frequency close to $f / 2$ (Longuet-Higgins 1950; Kadri \& Stiassnie 2013). The multiple-scale solution of $\S 3.2$ allows us to gain a deep physical insight on the system dynamics. As an example, consider the slowly varying bottom profile $h(x)=3000+1000 \tanh \left(2 \times 10^{-5} x\right)$, in metres, and a train of incident HA waves with frequency $f=0.2 \mathrm{~Hz}$. This value lies within the characteristic interval of HA frequencies in the deep ocean, see Kadri \& Stiassnie (2013). The water depth in the far field is $h_{\infty}=\lim _{x \rightarrow \infty} h(x)=4000 \mathrm{~m}$, to which corresponds a horizontal index of refraction $k_{n}\left(h_{\infty}\right)=7.528 \times 10^{-4} \mathrm{~m}^{-1}$ for the first mode $n=1$ (see again 2.20). Hence the wavelength along the direction of propagation in the far field is $\lambda_{n}\left(h_{\infty}\right)=2 \pi / k_{n}\left(h_{\infty}\right)=$ $8346 \mathrm{~m}$, which is a typical value for HA waves propagating in the deep ocean (Kadri \& Stiassnie 2013). We choose the constant longshore wavenumber $\alpha_{n}=5 \times 10^{-4} \mathrm{~m}^{-1}$. Note that this choice affects only the direction of propagation of the HA wave and the location of the critical depth. With the chosen $\alpha_{n}$, we get $\mu_{n}\left(h_{\infty}\right)=5.628 \times 10^{-4} \mathrm{~m}^{-1}$ for the 
(a)
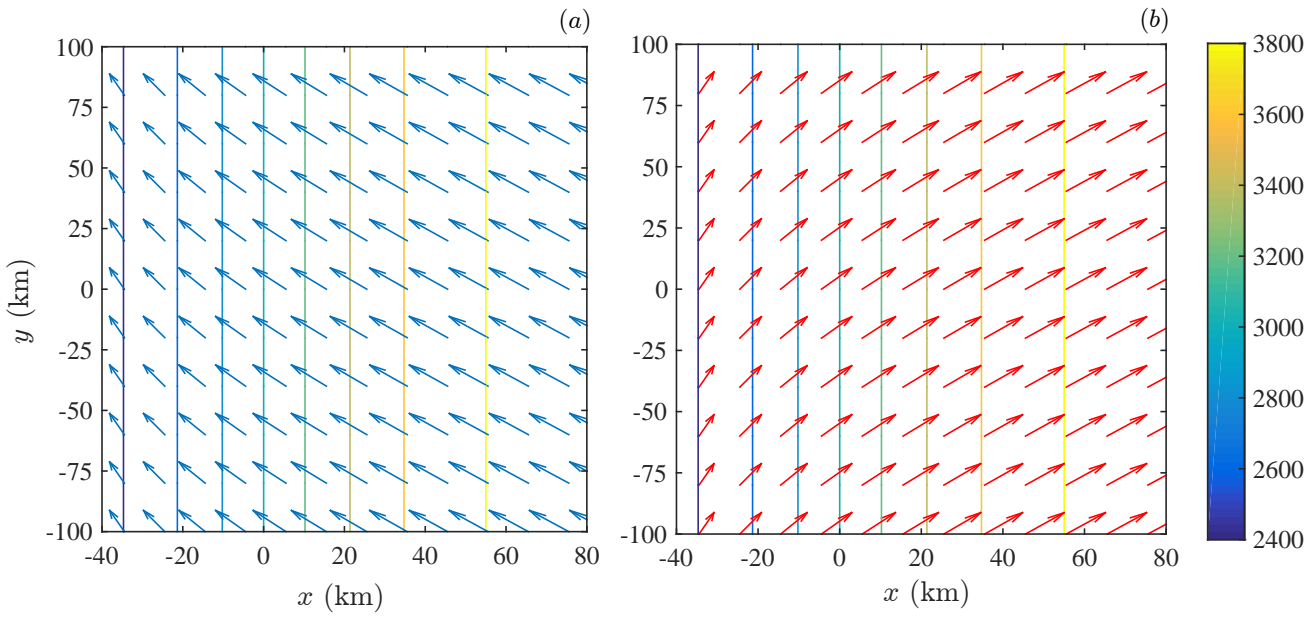

Figure 1. Contour plots of the bottom depth $h=3000+1000 \tanh \left(2 \times 10^{-5} x\right)$, in metres, and vector plots of the wavenumber vector (3.18). (a) Incoming wave: $\boldsymbol{k}_{n}^{-}(x)$, (b) Refracted wave: $\boldsymbol{k}_{n}^{+}(x)$. The parameters are $n=1, f=0.2 \mathrm{~Hz}$ and $\alpha_{n}=5 \times 10^{-4} \mathrm{~m}^{-1}$, which correspond to an angle of incidence of $132^{\circ}$.

offshore wavenumber (3.17) and $\theta_{n}^{-}\left(h_{\infty}\right) \sim 132^{\circ}$ for the angle of propagation (3.19) of the HA wave incoming from the far field. The critical depth is $d_{n}=2289 \mathrm{~m}$ and occurs at the turning point $x_{n}=-44.5 \mathrm{~km}$, where $\mu_{n}\left(x_{n}\right)=0$.

Let us first analyse the behaviour of the incoming HA wave away from the turning point. Figure 1 $(a)$ shows the contour plot of the bottom depth profile together with the vector plot of the wavenumber vector $\boldsymbol{k}_{n}^{-}(x)=\left\{-\mu_{n}(x), \alpha_{n}\right\}$ for the incoming HA wave, for the first mode $n=1$. Offshore of the turning point $\left(x>x_{n}\right)$, the water depth is greater than the critical depth $\left(h>d_{n}\right)$ and $\mu_{n}^{2}(x)>0$, see (3.30). The wave field is oscillatory, the wavenumber vector $\boldsymbol{k}_{n}^{-}$is oriented at about $132^{\circ}$ with respect to the $x$ axis (see right side of figure $1 a$ ), with the incoming HA crests normal to $\boldsymbol{k}_{n}^{-}$. Moving to shallower water towards the turning point $\left(x \rightarrow x_{n}\right)$, the water depth tends to the critical depth $\left(h \rightarrow d_{n}\right)$ and the offshore wavenumber of the incoming wave $\mu_{n} \rightarrow 0$, see (3.30). However, the longshore wavenumber $\alpha_{n}$ keeps constant following Snell's law (3.15). As a result, $\boldsymbol{k}_{n}^{-} \rightarrow\left\{0, \alpha_{n}\right\}$ as $h \rightarrow d_{n}$, i.e. $\boldsymbol{k}_{n}^{-}$decreases in magnitude and becomes increasingly parallel to the contours as the water depth decreases (see left side of figure $1 a$ ). Because of (3.14), the crests become increasingly orthogonal to the depth contours. Note that this refraction dynamics is opposite to the refraction of gravity waves, where crests become increasingly parallel to the contours as $h$ decreases, see Mei et al. (2005). At the turning point $\left(x=x_{n}\right)$ the incoming HA wave is refracted back to deeper water. The refracted wave is outgoing towards the far field and follows the pattern shown in figure $1(b)$ for $\boldsymbol{k}_{n}^{+}$, exiting the domain at an angle $\theta_{n}^{+} \sim 42^{\circ}$ with respect to the $x$ axis. Clearly, the total HA wave field in the fluid domain is made up by the sum of incoming and refracted wave components.

Close to the turning point $x_{n}=-44.5 \mathrm{~km},(3.18)$ ceases to be valid. The behaviour of the HA waves must be analysed with the uniform asymptotic solution (3.33). Figure 2 shows a section of the sample bottom depth profile (figure $2 a$ ), together with the behaviour of the slowly varying wavenumber $\mu_{n}$ (figure $2 b$ ) and the uniform asymptotic solution $\phi_{n}(x, 0)=X_{n}(x)$ (figure $2 c$ ), for $n=1$, over a long range along the offshore 

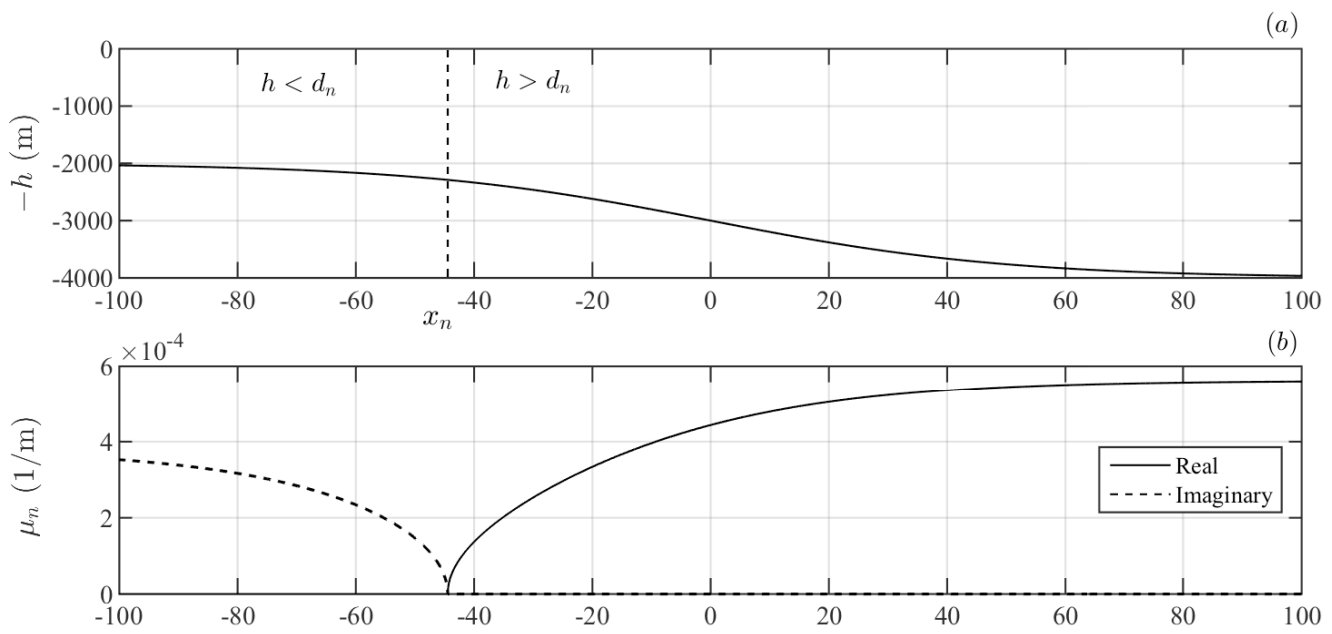

(c)

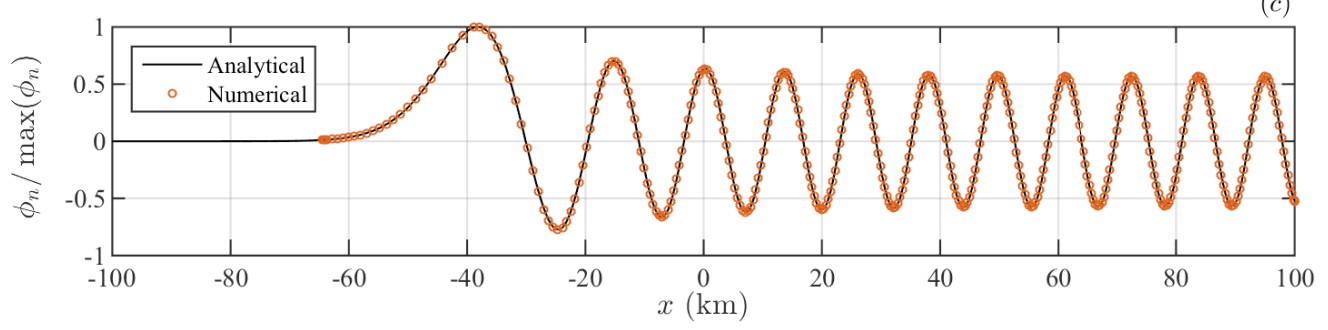

Figure 2. (a) Bottom profile (solid line) and critical depth (vertical dashed line), (b) Real (-) and imaginary (- -) part of the slowly varying wavenumber $\mu_{n}$, expression (3.30). (c) Normalised mode $\phi_{n} / \max \left(\phi_{n}\right)$ for $y=0$, see (3.33). Parameters are $n=1, f=0.2 \mathrm{~Hz}$ and $\alpha_{n}=0.0005 \mathrm{~m}^{-1}$.

axis $x$. Physically, the $\phi_{n}$ are exponentially decaying HA disturbances when the water depth is less than the critical depth $\left(h<d_{n}\right.$ for $\left.x<x_{n}\right)$, and the sum of incoming and refracted waves when the water depth is greater than the critical depth $\left(h>d_{n}\right.$ for $x>x_{n}$ ), as already shown in figure 1. Finally, we compared the asymptotic solution (3.33) to a fifth-order Runge-Kutta numerical solution of the governing equation (3.2), see figure 2(c). The agreement between the analytical approximation and the numerical results is excellent.

Depth-dependent evanescent/propagating dynamics were also observed in 2D by Kadri \& Stiassnie (2013) and Kadri (2015). However, the results obtained in this paper are new for two main reasons. Mathematically, unlike Kadri \& Stiassnie (2013)'s non-uniform result, the uniform approximation (3.33) seamlessly connects the near and far fields, without the existence of a transition zone where the solution is not unique (see figure 2 of Kadri \& Stiassnie 2013). Physically, the 3D propagation dynamics is significantly richer than in $2 \mathrm{D}$. In $2 \mathrm{D}$, the topography of the seafloor controls the shoaling effects, by which the HA waves change height and group velocity as they propagate towards the shallows. Because of such effects, the $n$th group velocity becomes null at the critical depth

$$
d_{n}^{(2 D)}=\frac{(2 n-1) \pi c}{2 \omega},
$$

causing complete reflection of the relevant HA mode (Kadri \& Stiassnie 2013). Indeed, 

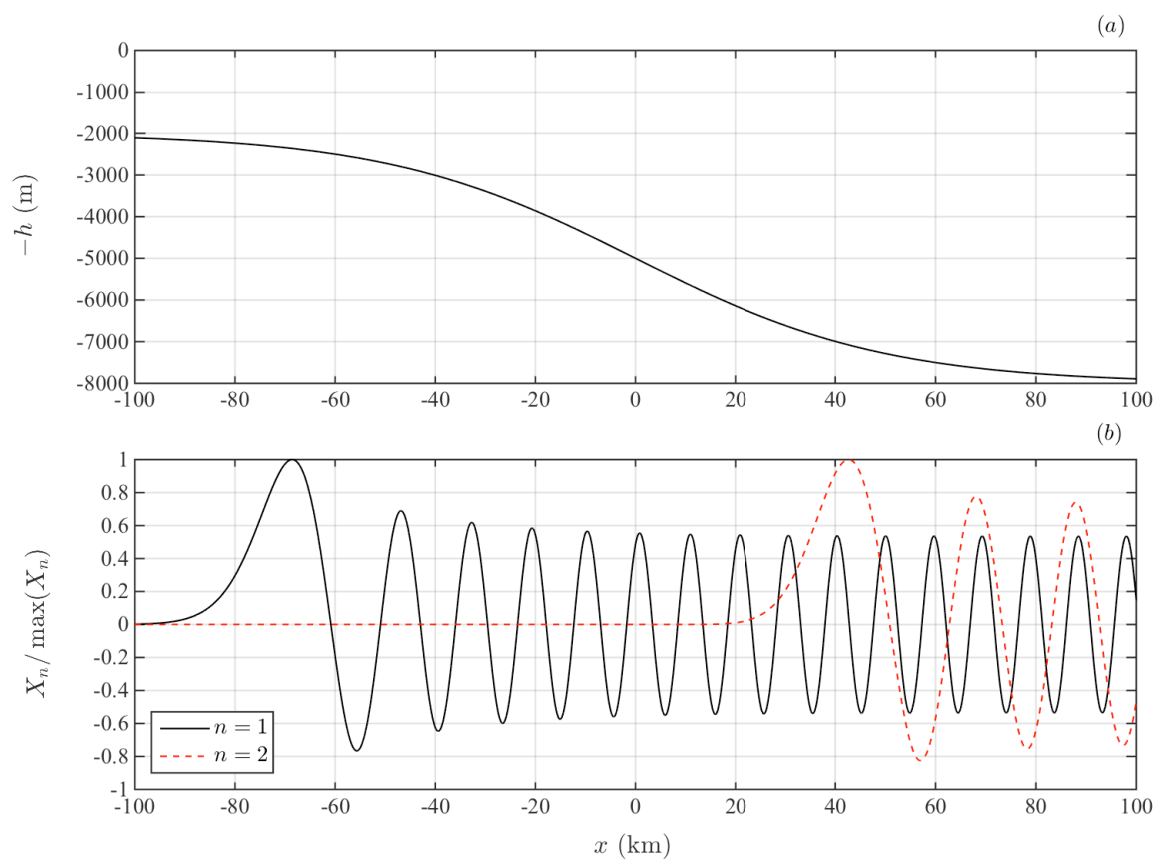

Figure 3. (a) Bottom profile for $h(x)=5000+3000 \tanh \left(2 \times 10^{-5} x\right)$, in metres, $(b)$ Normalised HA modes for $y=0$, see (3.33). Parameters are $f=0.2 \mathrm{~Hz}$ and $\alpha_{n}=0.0005 \mathrm{~m}^{-1}$.

the same result is obtained from (3.29) in the $2 \mathrm{D}$ limit $\alpha_{n}=0$. In $3 \mathrm{D}$, on the other hand, the topography of the seafloor controls both shoaling and refraction effects. As a consequence, the HA waves change both height and direction as they propagate towards shallow water (see again figure 1 and figure 2). Hence, in 3D each HA mode is not simply reflected back; instead, it gradually turns away as it approaches the shoreline (see figure 1a), until the wavenumber component normal to the shoreline vanishes at the relevant critical depth $d_{n}$, see (3.29). The latter defines the location where the $n$th mode is refracted down the shelf. Unlike the $2 \mathrm{D}$ limit (3.35), the critical depth in $3 \mathrm{D}$ (3.29) depends not only on the angular frequency $\omega$ of the incoming HA wave, but also on its longshore wavenumber $\alpha_{n}$. Expression (3.29) shows that the critical depth $d_{n}$ is minimum when $\alpha_{n}=0$ (normal incidence, 2D limit) and increases with increasing $\alpha_{n}$ (oblique incidence). Physically, the larger the longshore component of the incoming HA wave, the less it penetrates towards the shore.

Concerning the higher-order HA modes, note that the critical depth (3.29) increases linearly with the modal order $n$. Hence, higher-order modes are associated with greater critical depths and so propagate less onshore, as shown in figure 3 . The latter shows the plot of the sample bottom depth $h(x)=5000+3000 \tanh \left(2 \times 10^{-5} x\right)$ (figure $3 a$ ) together with the uniform asymptotic solutions $\phi_{n}(x, 0)=X_{n}(x)$ for the first 2 HA modes (figure $3 b)$. The higher the modal order, the earlier the incoming HA mode is refracted down to deep water and the less it penetrates into shallower water.

The refraction dynamics modelled by the MSEHA is in agreement with recent field measurements and numerical modelling of propagating HA waves in complex marine environments. As an example, Ballard (2012) has investigated the horizontal refraction 
of low-frequency sound off the southeast coast of Florida. Ballard (2012) has shown that, despite the sediment properties and the water-column sound-speed field exhibit significant range dependence over the shelf, the dynamics is almost exclusively controlled by the topography of the seafloor. Indeed, Ballard (2012)'s results confirm that lower modes propagate further up the shelf and the location where they are refracted down to deep water is controlled by their critical depth (see again figure 3 ).

\section{Forced hydro-acoustic frequencies over a slowly varying bottom}

\subsection{Analytical solution on a plane beach}

Building on the free-wave results, let us now study how single HA frequencies, forced by a tsunamigenic disturbance at the bottom of the ocean, propagate over range dependent environments. For the sake of example, consider the case of a plane beach of depth $h=\epsilon x$, with $\epsilon \ll 1$. Water is in the region $x>0$, the shoreline is at $x=0$. The planebeach geometry has important practical applications in tsunami models (Mei et al. 2005; Sammarco \& Renzi 2008). It is also particularly interesting as it allows one to obtain an exact analytical solution in terms of integrals of Bessel functions. Following Mei et al. (2005), consider a tsunamigenic bottom deformation, localised at $x=x_{0}$ :

$$
\bar{H}(\boldsymbol{x})=H_{0} \delta\left(\frac{x-x_{0}}{a}\right) \delta\left(\frac{y-0}{b}\right) .
$$

In the latter, $\delta$ is the delta function, $x_{0}>0$ is the position of the centre of the perturbation along the $x$ axis, $H_{0}$ is the magnitude of the deformation, $a$ and $b$ are non-dimensionalising parameters. Results for generic disturbances can be found via the Green theorem (Mei et al. 2005). Symmetry of the perturbation (4.1) with respect to the $x$ axis allows us to use the cosine Fourier transform pair along the shoreline

$$
\hat{\phi}_{n}(x ; \alpha)=\int_{0}^{\infty} \phi_{n}(x, y) \cos (\alpha y) \mathrm{d} y ; \quad \phi_{n}(x, y)=\frac{2}{\pi} \int_{0}^{\infty} \hat{\phi}_{n}(x ; \alpha) \cos (\alpha y) \mathrm{d} \alpha,
$$

so that the MSEHA (2.19) becomes

$$
\hat{\phi}_{n_{x x}}+\left[\frac{\omega^{2}}{c^{2}}-\alpha^{2}-\frac{(2 n-1)^{2} \pi^{2}}{4 \epsilon^{2} x^{2}}\right] \hat{\phi}_{n}=-\frac{4}{\pi} \frac{(-1)^{n}}{2 n-1} \mathrm{i} \omega H_{0} b \delta\left(\frac{x-x_{0}}{a}\right) \sqrt{\frac{2}{\epsilon x}} .
$$

We shall now solve (4.3) with the method of patched matching (Bender \& Orszag 1999). First, request that the solution be continuous at $x=x_{0}$ :

$$
\hat{\phi}_{n}\left(x_{0}+0 ; \alpha\right)=\hat{\phi}_{n}\left(x_{0}-0 ; \alpha\right) .
$$

Then, integrate (4.3) with respect to $x$ across the singularity at $x=x_{0}$, to get

$$
\hat{\phi}_{n_{x}}\left(x_{0}+0 ; \alpha\right)-\hat{\phi}_{n_{x}}\left(x_{0}-0 ; \alpha\right)=-\frac{4}{\pi} \frac{(-1)^{n}}{2 n-1} \mathrm{i} \omega H_{0} a b \sqrt{\frac{2}{\epsilon x_{0}}} .
$$

The governing equation (4.3) is of the Bessel kind (Mei 1997). The solution of (4.3) that satisfies the matching conditions (4.4)-(4.5) depends on the sign of $\alpha-\omega / c$. Again, we have two cases:

(i) $\alpha>\omega / c$. The solution is

$$
\hat{\phi}_{n}(x ; \alpha)=\frac{4 \sqrt{2}}{\pi} \frac{(-1)^{n}}{2 n-1} \mathrm{i} \omega H_{0} \frac{a b}{\epsilon} \sqrt{\epsilon x} \mathrm{~K}_{\nu_{n}}\left(\sqrt{\alpha^{2}-\frac{\omega^{2}}{c^{2}}} x_{0}\right) \mathrm{I}_{\nu_{n}}\left(\sqrt{\alpha^{2}-\frac{\omega^{2}}{c^{2}}} x\right), \quad x<x_{0},
$$


for all points landward of the bottom disturbance, and

$$
\hat{\phi}_{n}(x ; \alpha)=\frac{4 \sqrt{2}}{\pi} \frac{(-1)^{n}}{2 n-1} \mathrm{i} \omega H_{0} \frac{a b}{\epsilon} \sqrt{\epsilon x} \mathrm{I}_{\nu_{n}}\left(\sqrt{\alpha^{2}-\frac{\omega^{2}}{c^{2}}} x_{0}\right) \mathrm{K}_{\nu_{n}}\left(\sqrt{\alpha^{2}-\frac{\omega^{2}}{c^{2}}} x\right), \quad x>x_{0},
$$

for all points offshore of the disturbance. In (4.6) and (4.7), $\mathrm{I}_{\nu_{n}}$ and $\mathrm{K}_{\nu_{n}}$ are, respectively, the modified Bessel functions of first and second kind and order $\nu_{n}$, where

$$
\nu_{n}=\sqrt{\frac{1}{4}+\frac{(2 n-1)^{2} \pi^{2}}{4 \epsilon^{2}}} \sim \frac{(2 n-1) \pi}{2 \epsilon},
$$

for $\epsilon \ll 1$. Recall that $\mathrm{I}_{\nu_{n}}(z) \sim(0.5 z)^{\nu_{n}} / \Gamma\left(\nu_{n}+1\right)$ as $z \rightarrow 0$ and $\mathrm{K}_{\nu_{n}}(z) \sim$ $\sqrt{\pi /(2 z)} \exp (-z)$ as $z \rightarrow \infty$, see Olver et al. (2010). As a consequence, (4.6) yields $\hat{\phi}_{n} \rightarrow 0$ for $x \rightarrow 0$ and (4.7) gives $\hat{\phi}_{n} \rightarrow 0$ for $x \rightarrow \infty$. Expressions (4.6)-(4.7) represent trapped HA waves that are important only in a strip near the bottom deformation.

(ii) $\alpha<\omega / c$. The solution is

$$
\hat{\phi}_{n}(x ; \alpha)=-2 \sqrt{2} \frac{(-1)^{n}}{2 n-1} \omega H_{0} \frac{a b}{\epsilon} \sqrt{\epsilon x} \mathrm{H}_{\nu_{n}}\left(\sqrt{\frac{\omega^{2}}{c^{2}}-\alpha^{2}} x_{0}\right) \mathrm{J}_{\nu_{n}}\left(\sqrt{\frac{\omega^{2}}{c^{2}}-\alpha^{2} x}\right), \quad x<x_{0},
$$

for all points landward of the bottom disturbance, and

$$
\hat{\phi}_{n}(x ; \alpha)=-2 \sqrt{2} \frac{(-1)^{n}}{2 n-1} \omega H_{0} \frac{a b}{\epsilon} \sqrt{\epsilon x} \mathrm{~J}_{\nu_{n}}\left(\sqrt{\frac{\omega^{2}}{c^{2}}-\alpha^{2}} x_{0}\right) \mathrm{H}_{\nu_{n}}\left(\sqrt{\frac{\omega^{2}}{c^{2}}-\alpha^{2} x}\right), \quad x>x_{0},
$$

for all points offshore of the disturbance. In (4.9) and (4.10), $\mathrm{J}_{\nu_{n}}$ and $\mathrm{H}_{\nu_{n}}$ are, respectively, the Bessel and the Hankel functions of the first kind. Recall that $\mathrm{J}_{\nu_{n}}(z) \sim(0.5 z)^{\nu_{n}} / \Gamma\left(\nu_{n}+\right.$ 1) as $z \rightarrow 0$, see Olver et al. (2010). As a consequence, (4.9) yields $\hat{\phi}_{n} \rightarrow 0$ for $x \rightarrow 0$, i.e. $\hat{\phi}_{n}$ decays towards the shoreline. On the other hand, the function $\mathrm{H}_{\nu_{n}}$ in (4.10) is outgoing as $x \rightarrow \infty$. Therefore, (4.9)-(4.10) describe HA waves that decay near the shoreline and propagate towards the far field. Finally, substitution of (4.6)-(4.10) inside the inverse cosine Fourier transform (4.2), together with the decomposition (2.8), yields the $n$-th spatial HA potential

$$
\bar{\Phi}_{n}(\boldsymbol{x}, z)=\bar{\Phi}_{n}^{t}(\boldsymbol{x}, z)+\bar{\Phi}_{n}^{p}(\boldsymbol{x}, z) .
$$

In the latter,

$$
\begin{aligned}
\bar{\Phi}_{n}^{t}(\boldsymbol{x}, z) & =\frac{16}{\pi^{2}} \frac{(-1)^{n}}{2 n-1} \mathrm{i} \omega H_{0} \frac{a b}{\epsilon} \cos \left[(2 n-1) \frac{\pi}{2}\left(1+\frac{z}{\epsilon x}\right)\right] \\
& \times \int_{\omega / c}^{\infty}\left[\mathrm{K}_{\nu_{n}}\left(\sqrt{\alpha^{2}-\frac{\omega^{2}}{c^{2}}} x_{0}\right) \mathrm{I}_{\nu_{n}}\left(\sqrt{\alpha^{2}-\frac{\omega^{2}}{c^{2}}} x\right) H_{e}\left(x_{0}-x\right)\right. \\
& \left.+\mathrm{I}_{\nu_{n}}\left(\sqrt{\alpha^{2}-\frac{\omega^{2}}{c^{2}}} x_{0}\right) \mathrm{K}_{\nu_{n}}\left(\sqrt{\alpha^{2}-\frac{\omega^{2}}{c^{2}}} x\right) H_{e}\left(x-x_{0}\right)\right] \cos (\alpha y) \mathrm{d} \alpha
\end{aligned}
$$

is the trapped component, where $H_{e}$ is the Heaviside step function, while

$$
\bar{\Phi}_{n}^{p}(\boldsymbol{x}, z)=-\frac{8}{\pi} \frac{(-1)^{n}}{2 n-1} \omega H_{0} \frac{a b}{\epsilon} \cos \left[(2 n-1) \frac{\pi}{2}\left(1+\frac{z}{\epsilon x}\right)\right]
$$




$$
\begin{aligned}
& \times \int_{0}^{\omega / c}\left[\mathrm{H}_{\nu_{n}}\left(\sqrt{\frac{\omega^{2}}{c^{2}}-\alpha^{2}} x_{0}\right) \mathrm{J}_{\nu_{n}}\left(\sqrt{\frac{\omega^{2}}{c^{2}}-\alpha^{2}} x\right) H_{e}\left(x_{0}-x\right)\right. \\
& \left.+\mathrm{J}_{\nu_{n}}\left(\sqrt{\frac{\omega^{2}}{c^{2}}-\alpha^{2}} x_{0}\right) \mathrm{H}_{\nu_{n}}\left(\sqrt{\frac{\omega^{2}}{c^{2}}-\alpha^{2}} x\right) H_{e}\left(x-x_{0}\right)\right] \cos (\alpha y) \mathrm{d} \alpha
\end{aligned}
$$

is the propagating component. Hence the tsunamigenic bottom disturbance (4.1) generates a twofold HA wave field, made by trapped components (4.12) and progressive components which propagate offshore, but decay towards the shoreline (4.13). This happens precisely because the HA frequencies turn away as they approach the shore (see again §3). The dynamic pressure can be determined from the HA potential with the expression $p_{n}(\boldsymbol{x}, z, t)=-\rho_{0} \Phi_{n_{t}}$ (see Renzi \& Dias 2014a), which yields $p_{n}(\boldsymbol{x}, z, t)=$ $p_{n}^{t}(\boldsymbol{x}, z, t)+p_{n}^{p}(\boldsymbol{x}, z, t)$. In the latter,

$$
p_{n}^{(t, p)}(\boldsymbol{x}, z, t)=\operatorname{Re}\left\{\mathrm{i} \rho_{0} \omega \bar{\Phi}_{n}^{(t, p)}(\boldsymbol{x}, z) \mathrm{e}^{-\mathrm{i} \omega t}\right\}
$$

is the dynamic pressure of the $n$th trapped (propagating) HA component. Finally, we define the transmission loss as

$$
\mathrm{TL}=-20 \log _{10}\left|\frac{\bar{p}(\boldsymbol{x}, z)}{p_{0}(r=1 \mathrm{~m})}\right|,
$$

where $\bar{p}(\boldsymbol{x}, z)=\mathrm{i} \rho_{0} \omega \bar{\Phi}(\boldsymbol{x}, z)$ is the spatial component of the total pressure generated by the bottom disturbance and

$$
p_{0}(r)=\rho_{0} \omega^{2} H_{0} a b \frac{\exp (\mathrm{i} \omega r / c)}{4 \pi r}
$$

is the pressure produced at a distance $r$ by a source of the same intensity as the bottom disturbance, but in an infinite, homogeneous medium with the ambient density $\rho_{0}$ (Jensen et al. 2011).

\subsection{Numerical example}

Let us now analyse the propagation of HA waves generated by a tsunamigenic disturbance at the bottom of a plane beach. For simplicity, we shall consider a unit-volume displacement, i.e. $a=b=H_{0}=1 \mathrm{~m}$, so that the results will be per unit $\mathrm{m}^{3}$ of displaced volume. The bottom slope is $\epsilon=0.08$. Figure 4 shows the density plot of the pressure $p_{n}^{t}$ of the trapped HA component (4.14) of frequency $f=0.2 \mathrm{~Hz}$ and modal order $n=1$ at the bottom of the ocean, $z=-\epsilon x$, generated by the bottom disturbance at $x_{0}=25 \mathrm{~km}$ from the shoreline, at a depth $h_{0}=2 \mathrm{~km}$. This choice of parameters is consistent with the 1693 East Mediterranean earthquake scenario studied by Cecioni et al. (2015), in which the signal generated at a depth $h=2 \mathrm{~km}$ has a carrier frequency of $f \sim 0.2 \mathrm{~Hz}$. Figure 4 shows that the trapped component decays either onshore and offshore along the $x$ axis, but propagates parallel to the shoreline. Note that the trapped component is not refracted, because it has not a progressive-wave structure along $x$. The plot of the full dynamic pressure $p_{n}=p_{n}^{t}+p_{n}^{p}$ is shown in figure 5 . The signal mostly propagates from the generation point towards deeper water in the form of outgoing progressive waves. This means that the dynamics away from the source is dominated by the propagating component $p_{n}^{p}$, which is stronger than the trapped longshore component $p_{n}^{t}$ of figure 4 . Figure 5 also reveals that shorter HA waves travel faster, followed by a tail of longer waves (see also Yamamoto 1982; Renzi \& Dias 2014b). This is opposite to the dispersive 


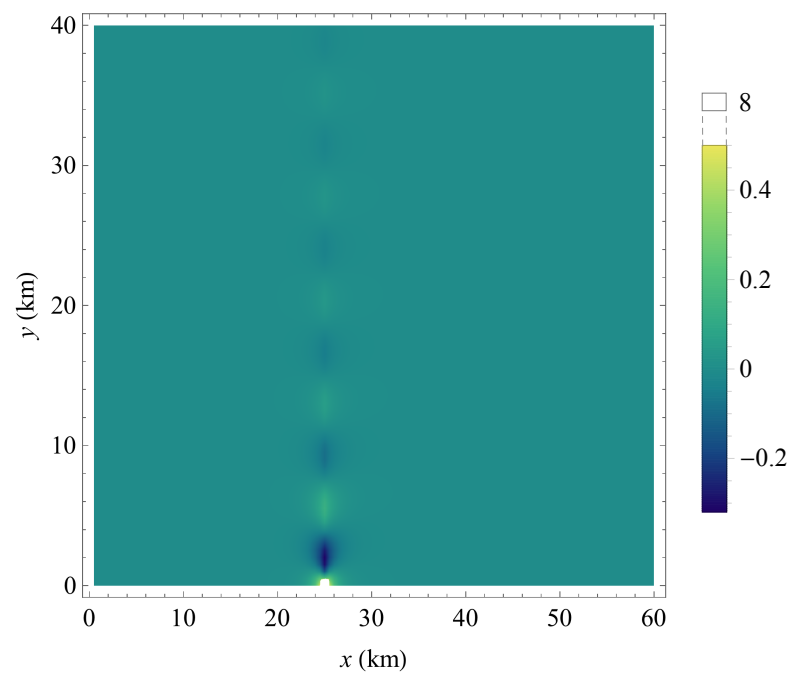

Figure 4. Pressure $p_{n}^{t}$ of the trapped HA component at the bottom of a plane beach, $z=-\epsilon x$, generated by a bottom disturbance located at $x_{0}=25 \mathrm{~km}$ from the shoreline; $x$ and $y$ denote the offshore and longshore directions, respectively. The parameters are $n=1, f=0.2 \mathrm{~Hz}, \epsilon=0.08$, $a=b=H_{0}=1 \mathrm{~m}$. Pressure values are in $\mathrm{Pa} / \mathrm{m}^{3}$ of displaced volume. Values are clipped near the source for easiness of reading.

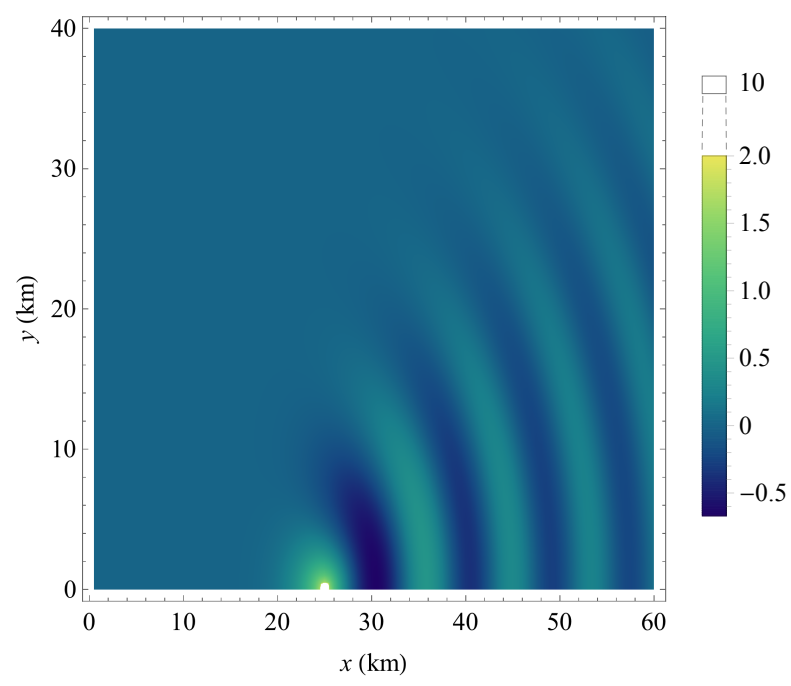

Figure 5. Dynamic pressure $p_{n}=p_{n}^{t}+p_{n}^{p}$ at the bottom of a plane beach, generated by a bottom disturbance at $x_{0}=25 \mathrm{~km}$. The parameters are the same as in figure 4 . Pressure values are in $\mathrm{Pa} / \mathrm{m}^{3}$ of displaced volume. Values are clipped near the source for easiness of reading.

behaviour of gravity waves (Mei et al. 2005). Finally, note that the refraction effects discussed in $\S 3$ limit the transmission of the signal towards the shore.

We have also analysed the behaviour of higher modes. Already for $n=2$, the dynamic pressure $p_{n}$ is noticeable only in a very small region near the source and then vanishes quickly away from it. This agrees with the results of Stiassnie (2010), who found that the first mode carries most of the energy transferred by the bottom motion to the HA waves. 


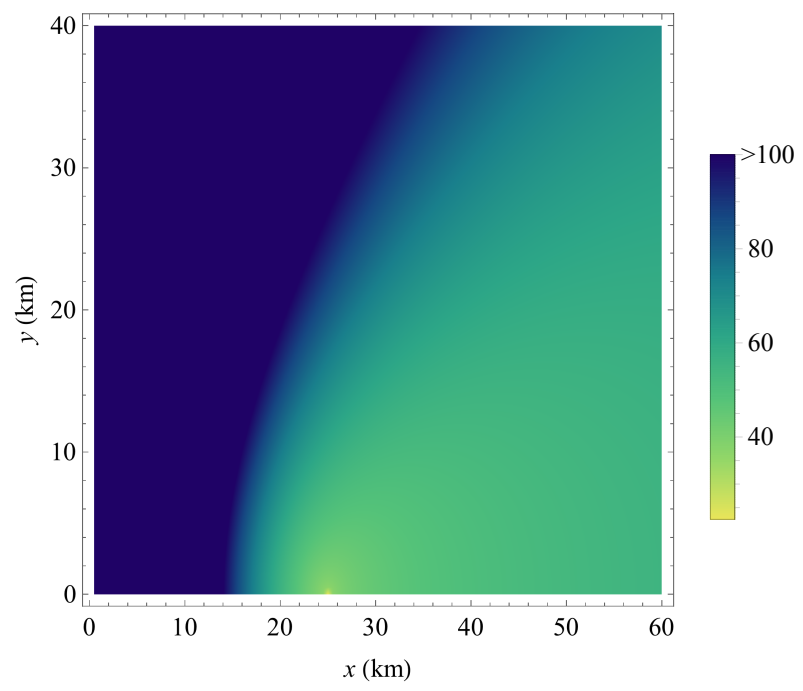

Figure 6 . Transmission loss TL (4.15) at the bottom of a plane beach generated by a bottom disturbance at $x_{0}=25 \mathrm{~km}$. The parameters are the same as in figure 4 . Transmission loss values are in $\mathrm{dB}$ re $1 \mathrm{~m}$.

Equally, Eyov et al. (2013), Hendin \& Stiassnie (2013), Kadri (2015) and Cecioni et al. (2015) showed that the first HA mode has the largest amplitude and contains most of the energy. As a consequence, the higher modes $n>1$ do not influence the transmission loss (4.15) away from the source.

Figure 6 shows the transmission loss at the bottom of the fluid domain, $z=-\epsilon x$. The plot highlights the existence of a shadow zone where TL is maximum and the signal is strongly attenuated. This result explains the interesting observations of Cecioni et al. (2015) on the numerical simulation of the AD 365 Eastern Mediterranean earthquake. Cecioni et al. (2015) noted that the HA perturbation generated by the earthquake off western Crete could not reach the shallower water areas south-west of Sicily, even after long time from the earthquake, despite the associated tsunami did hit Sicily. Cecioni et al. (2015) hypothesised a filtering effect of the water depth as the cause of the phenomenon. The analytical solution of the MSEHA further clarifies the reason of this dynamics: refraction turns the propagating HA wave frequencies down to deep water, preventing them from reaching the shallows, where indeed the transmission loss is maximum (see again figure 6).

\section{Conclusions}

We derived a normalised form of the weakly compressible mild-slope equation for HA waves in 3D (Sammarco et al. 2013) and showed that it satisfies the conservation of energy on a slowly varying bottom. We obtained a novel uniform analytical solution of the equation, based on a multiple-scale perturbation technique. For free HA waves over topography, the bathymetry of the seafloor controls the change in height and direction of incident HA waves as they propagate towards the shallows. Each HA mode gradually turns away from the shoreline until it is completely refracted back at the relevant critical depth. The extent to which the HA modes propagate onshore depends on their longshore wavenumber. The smaller the longshore component, the farther the incident 
HA wave penetrates in shallow water before being refracted back. Also, low-order modes are associated with smaller critical depths and so propagate farther onshore.

For forced generation by a tsunamigenic disturbance on a plane beach, we derived a novel exact analytical solution in terms of integrals of Bessel functions. We showed that the HA wave field is made up by a longshore trapped and an offshore propagating component, which is dominant away from the disturbance. Shorter waves travel faster, followed by a tail of longer waves. Refraction effects limit the transmission of the HA wave field towards the shore. As a consequence, there exists a shadow zone near the shoreline where the transmission loss is maximum and the signal vanishes. This result is particularly important, because TL plots like that of figure 6 can be used to determine the optimal position of hydrophone networks capable of capturing the HA signal generated by an underwater earthquake, in advance of the associated tsunami.

We remark that the bottom of the ocean has been assumed rigid in this study. Eyov et al. (2013) recently developed a model for an elastic bottom in 2D, which shows that neglecting the elasticity is justified far from the critical depth. At the critical depth, the leading mode turns into a Scholte wave, due to the modification of the group and phase speeds induced by the bottom elasticity with respect to the rigid case. Eyov et al. (2013)'s results would suggest that over a range dependent elastic bottom in 3D, HA wave refraction is governed by the bathymetry only at frequencies above the cut-off, with the exception of the first mode. This implies that physically refraction dominates all modes in deep water, whereas below the critical depth $\left(h<d_{n}\right)$ the present solution could be valid only for the first HA mode, which indeed is dominant. We further remark that mathematical solutions of HA waves generated by tsunamigenic disturbances over an elastic bottom in 3D appear not to be available in the literature, leaving this challenging issue as a topic for further research.

In this paper, we have shown that the trapped longshore HA waves have the remarkable property of existing at any water depth. For such waves, energy is confined near the source in the offshore direction and propagates along a narrow strip parallel to bathymetry. In the case of a bottom dislocation, those longshore waves are much smaller than the offshore propagating waves, which are dominant. This highlights the importance of deep-sea observatories to detect the HA signal generated by underwater earthquakes (Cecioni et al. 2015). However, in the case of a submerged landslide, the scenario could be different. Since HA waves are generated at different depths as the slide moves down the incline, energy is transmitted to them at several frequencies and modes. In this case, the trapped low-frequency HA component could be significant and longshore HA waves could be used to predict incoming edge waves propagating along a beach (Sammarco \& Renzi 2008). This intriguing research hypothesis is being considered in ongoing work.

E.R. acknowledges the suggestions of two anonymous referees that have contributed to the improvement of the paper.

\section{Appendix A. Hydro-acoustic energy flux}

Consider the $n$th HA mode and a vertical cross section of unit width orthogonal to the direction of propagation along the velocity vector $\boldsymbol{u}_{n}(\boldsymbol{x}, z, t)$. The rate of energy flux across the section is equal to the mean rate of work done by the dynamic pressure (Mei 
et al. 2005; Jensen et al. 2011)

$$
\mathcal{F}_{n}(\boldsymbol{x})=\frac{\omega}{2 \pi} \int_{0}^{\frac{2 \pi}{\omega}} \int_{-h(\boldsymbol{x})}^{0} p_{n}(\boldsymbol{x}, z, t) \boldsymbol{u}_{n}(\boldsymbol{x}, z, t) \mathrm{d} z \mathrm{~d} t
$$

In the latter, $p_{n}(\boldsymbol{x}, z, t)=-\rho \Phi_{n_{t}}$ is the $n$th order dynamic pressure and $\boldsymbol{u}_{n}(\boldsymbol{x}, z, t)=$ $\nabla \Phi_{n}$ is the horizontal velocity vector. The potential $\Phi_{n}(\boldsymbol{x}, z, t)=\operatorname{Re}\left\{\bar{\Phi}_{n}(\boldsymbol{x}, z) \mathrm{e}^{-\mathrm{i} \omega t}\right\}=$ $\operatorname{Re}\left\{\phi_{n}(\boldsymbol{x}) Z_{n}(\boldsymbol{x}, z) \mathrm{e}^{-\mathrm{i} \omega t}\right\}$, where the $Z_{n}$ are still given by (2.17). Performing the latter substitutions, (A 1) becomes

$$
\mathcal{F}_{n}(\boldsymbol{x})=\frac{\rho \omega}{2 \pi} \int_{-h}^{0} \int_{0}^{\frac{2 \pi}{\omega}} \operatorname{Re}\left\{\mathrm{i} \omega \phi_{n}(\boldsymbol{x}) Z_{n}(\boldsymbol{x}, z) \mathrm{e}^{-\mathrm{i} \omega t}\right\} \operatorname{Re}\left\{\nabla\left[\phi_{n}(\boldsymbol{x}) Z_{n}(\boldsymbol{x}, z)\right] \mathrm{e}^{-\mathrm{i} \omega t}\right\} \mathrm{d} z \mathrm{~d} t .
$$

Now recall the property

$$
\frac{\omega}{2 \pi} \int_{0}^{\frac{2 \pi}{\omega}} \operatorname{Re}\left\{A \mathrm{e}^{-\mathrm{i} \omega t}\right\} \operatorname{Re}\left\{B \mathrm{e}^{-\mathrm{i} \omega t}\right\} \mathrm{d} t=\frac{1}{2} \operatorname{Re}\left\{A^{*} B\right\}
$$

for any complex values $A$ and $B$ independent of $t$ (Mei 1997). Hence, use of (A 3) and the property $<Z_{n}, Z_{n}>=1$ transforms (A 2) into

$$
\mathcal{F}_{n}(\boldsymbol{x})=-\frac{\rho \omega}{2} \operatorname{Re}\left\{\mathrm{i} \phi_{n}^{*}(\boldsymbol{x})\left[\nabla \phi_{n}(\boldsymbol{x})+\phi_{n}(\boldsymbol{x}) \int_{-h}^{0} Z_{n}(\boldsymbol{x}, z) \nabla Z_{n}(\boldsymbol{x}, z) \mathrm{d} z\right]\right\} .
$$

Now recall that $Z_{n}(\boldsymbol{x}, z)=Z_{n}[h(\boldsymbol{x}), z]$, so that $\nabla Z_{n}(\boldsymbol{x}, z)=Z_{n_{h}}(h, z) \nabla h(\boldsymbol{x})$. Substitute the latter into (A 4$)$ and use the property $<Z_{n}(\boldsymbol{x}, z), Z_{n_{h}}(\boldsymbol{x}, z)>=-1 / h$ to obtain finally

$$
\mathcal{F}_{n}(\boldsymbol{x})=\frac{\rho \omega}{2} \operatorname{Im}\left\{\phi_{n}^{*}(\boldsymbol{x}) \nabla \phi_{n}(\boldsymbol{x})\right\}=\frac{\rho \omega}{2} \operatorname{Im}\left\{\phi_{n}^{*}(\boldsymbol{x}) \boldsymbol{u}_{n}(\boldsymbol{x})\right\}
$$

The latter is the sought HA energy flux vector, which is oriented along the direction of propagation of the perturbation, as expected.

\section{REFERENCES}

BALlard, M.S. 2012 Modeling three-dimensional propagation in a continental shelf environment. J. Acoust. Soc. Am. 131 (3), 1969-1977.

Bender, C. M. \& Orszag, S. A. 1999 Advanced Mathematical Methods for Scientists and Engineers. Springer.

Cecioni, C., Abdolali, A., Bellotti, G. \& Sammarco, P. 2015 Large-scale numerical modeling of hydro-acoustic waves generated by tsunamicenic earthquakes. Nat. Hazards Earth Syst. Sci. 15, 627-636.

Cheng, H. 2007 Advanced Analytic Methods in Applied Mathematics, Science and Engineering. Boston (USA): LuBan Press.

Dingemans, M.W. 1997 Water Wave Propagation over Uneven Bottoms. Singapore: World Scientific.

Eyov, E., Klar, A., Kadri, U. \& Stiassnie, M. 2013 Progressive waves in a compressible ocean with an elastic bottom. Wave Motion 50, 929-939.

Hendin, G. \& Stiassnie, M. 2013 Tsunami and acoustic-gravity waves in water of constant depth. Phys. Fluids 25, 086103.

Hildebrand, F.B. 1976 Advanced Calculus for Applications. New Jersey (USA): Prentice-Hall. Jensen, F.B., Kuperman, W.A., Porter, M.B. \& Schmidt, H. 2011 Computational Ocean Acoustics. New York (USA): Springer.

KADRI, U. 2015 Wave motion in a heavy compressible fluid: Revisited. Eur. J. Mech. B-Fluid 49, $50-57$. 
KADRI, U. \& Stiassnie, M. 2013 A note on the shoaling of acoustic-gravity waves. WSEAS transactions on Fluid Mechanics 8 (2), 43-49.

Longuet-Higgins, M.S. 1950 A theory on the origin of microseisms. Philos. Trans. R. Soc. London, Ser. A 243, 1-35.

Massel, S. R. 2013 Ocean Surface Waves. World Scientific.

MeI, C. C. 1997 Mathematical Analysis in Engineering. Cambridge (UK): Cambridge University Press.

Mei, C. C., Stiassnie, M. \& Yue, D. K.-P. 2005 Theory and Application of Ocean Surface Waves. Singapore: World Scientific.

Olver, F.W.J., Lozier, D.W., Boisvert, R.F. \& Clark, C.W. 2010 NIST Handbook of Mathematical Functions. Cambridge University Press.

RENZI, E. \& DiAs, F. 2014a Hydro-acoustic precursors of gravity waves generated by surface pressure disturbances localised in space and time. J. Fluid Mech. 754, 250-262.

Renzi, E. \& DiAs, F. $2014 b$ Time-domain hydroacoustic Green function for surface pressure disturbance. In Proceedings of the 29th Intl. Workshop on Water Waves and Floating Bodies, Osaka (Japan).

Sammarco, P., Cecioni, C., Bellotti, G. \& Abdolali, A. 2013 Depth-integrated equation for large-scale modelling of low-frequency hydroacoustic waves. J. Fluid Mech. 722 (R6), $1-10$.

SAmmarco, P. \& Renzi, E. 2008 Landslide tsunamis propagating along a plane beach. J. Fluid Mech. 598, 107-119.

Stiassnie, M. 2010 Tsunamis and acoustic-gravity waves from underwater earthquakes. J. Engng Maths 67, 23-32.

Yамамото, T. 1982 Gravity waves and acoustic waves generated by submarine earthquakes. Soil Dyn. Earthq. Eng. 1 (2), 75-82. 